\title{
BMJ Open Family-based interventions to increase physical activity in children: a meta-analysis and realist synthesis protocol
}

\author{
Helen Elizabeth Brown, ${ }^{1,2}$ Andrew J Atkin, ${ }^{2}$ Jenna Panter, ${ }^{1,2}$ Kirsten Corder, ${ }^{1,2}$ \\ Geoff Wong, ${ }^{3}$ Mai J M Chinapaw, ${ }^{4}$ Esther van Sluijs ${ }^{1,2}$
}

To cite: Brown HE, Atkin AJ, Panter J, et al. Family-based interventions to increase physical activity in children: a meta-analysis and realist synthesis protocol. BMJ Open 2014;4:e005439. doi:10.1136/bmjopen-2014005439

- Prepublication history for this paper is available online. To view these files please visit the journal online (http://dx.doi.org/10.1136/ bmjopen-2014-005439).

Received 9 April 2014 Revised 15 July 2014 Accepted 17 July 2014

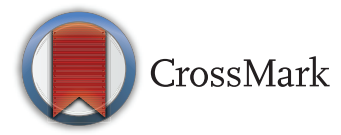

${ }^{1}$ Department of MRC Epidemiology, University of Cambridge School of Clinical Medicine, Institute of Metabolic Science, Cambridge, UK ${ }^{2}$ UKCRC Centre for Diet and Activity Research (CEDAR), Institute of Public Health, University of Cambridge, Cambridge, UK

${ }^{3}$ Centre for Primary Care and Public Health, University of London, London, UK ${ }^{4}$ Department of Public and Occupational Health, EMGO Institute for Health and Care Research, VU University Medical Centre, Amsterdam, The Netherlands

Correspondence to Dr Helen Elizabeth Brown; heb56@medschl.cam.ac.uk

\section{ABSTRACT}

Introduction: Despite the established relationship between physical activity and health, data suggest that many children are insufficiently active, and that levels decline into adolescence. Engaging the family in interventions may increase and maintain children's physical activity levels at the critical juncture before secondary school. Synthesis of existing evidence will inform future studies, but the heterogeneity in target populations recruited, behaviour change techniques and intervention strategies employed, and measurement conducted, may require a multifaceted review method. The primary objective of this work will therefore be to synthesis evidence from intervention studies that explicitly engage the family unit to increase children's physical activity using an innovative dual meta-analysis and realist approach.

Methods and analysis: Peer-reviewed studies will be independently screened by two authors for inclusion based on (1) including 'healthy' participants aged 5-12 years; (2) having a substantive intervention aim of increasing physical activity, by engaging the family and (3) reporting on physical activity. Duplicate data extraction and quality assessment will be conducted using a specially designed proforma and the Effective Public Health Practice Project Quality Assessment Tool respectively. STATA software will be used to compute effect sizes for meta-analyses, with subgroup analyses conducted to identify moderating characteristics. Realist syntheses will be conducted according to RAMESES quality and publication guidelines, including development of a programme theory and evidence mapping.

Dissemination: This review will be the first to use the framework of a traditional review to conduct a dual meta-analysis and realist synthesis, examining interventions that engage the family to increase physical activity in children. The results will be disseminated through peer-reviewed publications, conferences, formal presentations to policy makers and practitioners and informal meetings. Evidence generated from this synthesis will also be used to inform the development of theory-driven, evidencebased interventions aimed at engaging the family to increase physical activity levels in children.
Protocol registration: International Prospective Register for Systematic Reviews (PROSPER0): number CRD42013005780.

\section{BACKGROUND}

The relationship between physical activity and health is well established; in children, physical activity is associated with cardiovascular risk factors, ${ }^{12}$ body composition (particularly waist circumference and fat mass $)^{34}$ and bone health. ${ }^{5}$ Engaging regularly in physical activity has a beneficial effect on depression, anxiety and self-esteem, and may also be associated with improved cognitive performance and scholastic achievement in this age group. ${ }^{6}$ Despite this, data from several countries suggest that the majority of children are insufficiently active to confer health benefit ${ }^{78}$; and that levels of physical activity decline substantially throughout childhood and into adolescence. ${ }^{9} 10$

Intervention prior to this age-related decline (commonly between 9 and 11 years of age) may be important to maintain adequate physical activity levels. Observational evidence suggests that a steeper decline in physical activity occurs out of school time, particularly at the weekend, ${ }^{11}$ and so intervention during these periods may represent the most responsible use of limited public health resources. ${ }^{12}$ However, the school environment is the most frequently used setting for researchers trying to improve children's physical activity patterns; as such, studies have been largely unsuccessful. ${ }^{13} 14$

A recent National Institute for Health and Care Excellence review identified characteristics of successful interventions, highlighting 
those based in the home as most effective in changing health behaviours. ${ }^{15}{ }^{16}$ Parental support has been consistently and positively associated with increased physical activity in children. ${ }^{17}$ The addition of a family component (eg, parent education) to school-based interventions has also proved to be efficacious. ${ }^{14}$ Engaging the family may therefore be a promising strategy, and may support the maintenance of physical activity levels (and concurrently avoid an increase in sedentary behaviour) at the critical juncture before children progress to secondary school. ${ }^{14} 18$

Currently, only one systematic overview with a focus on family-based interventions is available. O'Connor et $a l^{19}$ reviewed literature published before January 2008, concluding that the large number of pilot studies, and the variability in study design and outcome measures, restricted insight into how best to engage parents in physical activity promotion. Other broader reviews have included subsections on family-based interventions, reaching similar conclusions. ${ }^{18} 20$ To develop effective interventions, a more thorough understanding of such studies is required. A brief scoping review identified a number of interventions published within the last 5 years, indicating the need for an update in this field. This may include mediation analysis to explore causal mechanisms, and process evaluation to examine the implementation, receipt and setting of an intervention and support interpretation of results. ${ }^{21}$

A synthesis of existing evidence would inform future family-based studies aimed at improving and maintaining physical activity levels in children. However, the heterogeneity in target populations recruited, behaviour change techniques and intervention strategies employed, measurement conducted and number of pilot studies included, may require a multifaceted approach. Well-conducted meta-analyses allow for an objective appraisal of the evidence, provide a precise estimate of a treatment effect, and may explain heterogeneity between the results of individual studies. ${ }^{22} 23$ Meta-analytic techniques are, however, limited to quantitative analysis, which may not be sufficient to understand how an intervention operates. In contrast, a realist synthesis offers no quantifiable summary of intervention effectiveness, but considers the interaction between context, mechanism and outcome, exploring 'what works for whom, under what circumstances, how and why? ${ }^{24}$ Previous reviews in this area have been somewhat constrained by these limitations, and therefore provide insufficient insight into the complex causal pathways that may underpin interventions.

Combining these two methodologies, using the framework of a traditional systematic review, may provide a more comprehensive examination of studies. The primary objective of this work will therefore be to summarise, using the innovative dual meta-analysis and realist synthesis approach, existing peer-reviewed intervention studies that explicitly engage the family unit to increase physical activity in children.

\section{METHODS}

The protocol is registered with the International Prospective Register for Systematic Reviews (PROSPERO) CRD42013005780.

Given the multifaceted approach planned, the methods for this work will be divided by process. The first section will detail overall review methods that relate to all studies, including search strategy, study screening, inclusion and exclusion criteria, quality assessment and general data extraction. The second section, headed Realist Synthesis, will outline data extraction and analysis procedures specific to the realist synthesis. The third section, headed Meta-analysis, will provide complementary information related to the meta-analysis.

\section{Search strategy}

The following databases will be searched for articles published up to and including June 2013, with no limit on earliest year of publication: PubMed (title and abstract), Web of Knowledge (topic), Scopus (title, abstract and keywords), Ovid MEDLINE (abstract), PsycInfo (abstract). The search strategy will be common across databases, and will consist of PICO terms; participants of interest, intervention, control and primary outcome of interest. Full details of search terms used will be reported using figure 1.

In addition to database searches, reference lists of included full-text articles and personal archives will be screened for studies meeting inclusion criteria. Four review articles, focusing on interventions in a range of settings, have been identified and will also be used to source additional studies. ${ }^{14}$ 18-20

\section{Study screening}

All retrieved titles and abstracts will be screened by the primary author (HEB), and duplicate screened by another author (AJA or EvS). Criteria for screening will be refined if necessary, and any discrepancy in inclusion or exclusion will be resolved through a consensus discussion among the three authors (HEB, EvS and AJA). Full text versions of selected articles will then be obtained, and inclusion and exclusion criteria assessed (following the same procedure as for titles and abstracts; duplicate screening and consensus discussion between HEB, EvS and AJA).

\section{Inclusion and exclusion criteria}

Studies will be included that report on interventions that (1) have an explicit aim to increase physical activity, (2) use the family as the setting, (3) include healthy children aged 5-13 years at baseline, (4) report on results from a valid measure of physical activity and (5) were published in a peer-reviewed journal up to and including June 2013. Study designs may include randomised controlled trials, comparison trials and/or quasiexperimental studies. Irrespective of the over-arching aim (eg, to reduce weight), if a substantive part of the intervention targets the determinants of physical activity, or aims to 
Databases: PubMed, Web of Knowledge, Scopus, Medline, Psyclnfo

Search terms

Population: children OR child OR childhood OR adolescence OR adolescent OR teen OR teenage OR teenager OR teens OR teenagers OR teenaged OR "young people" OR youth OR youths OR boy OR girl OR "school - aged"

AND

Study design: intervention OR trial OR randomised OR controlled OR comparison OR experiment OR "quasi-experimental" OR cluster

AND

Intervention type: family OR "family-based" OR parent OR mother OR father OR "primary care-giver" OR guardian OR sibling OR brother OR sister OR home OR "home-based"

AND

Behaviour: "physical activity" OR "physical activities" OR exercise OR "energy expenditure" OR sport OR sports OR "active travel" OR "walking" OR "cycling"

The following MeSH terms will be used in PubMed:
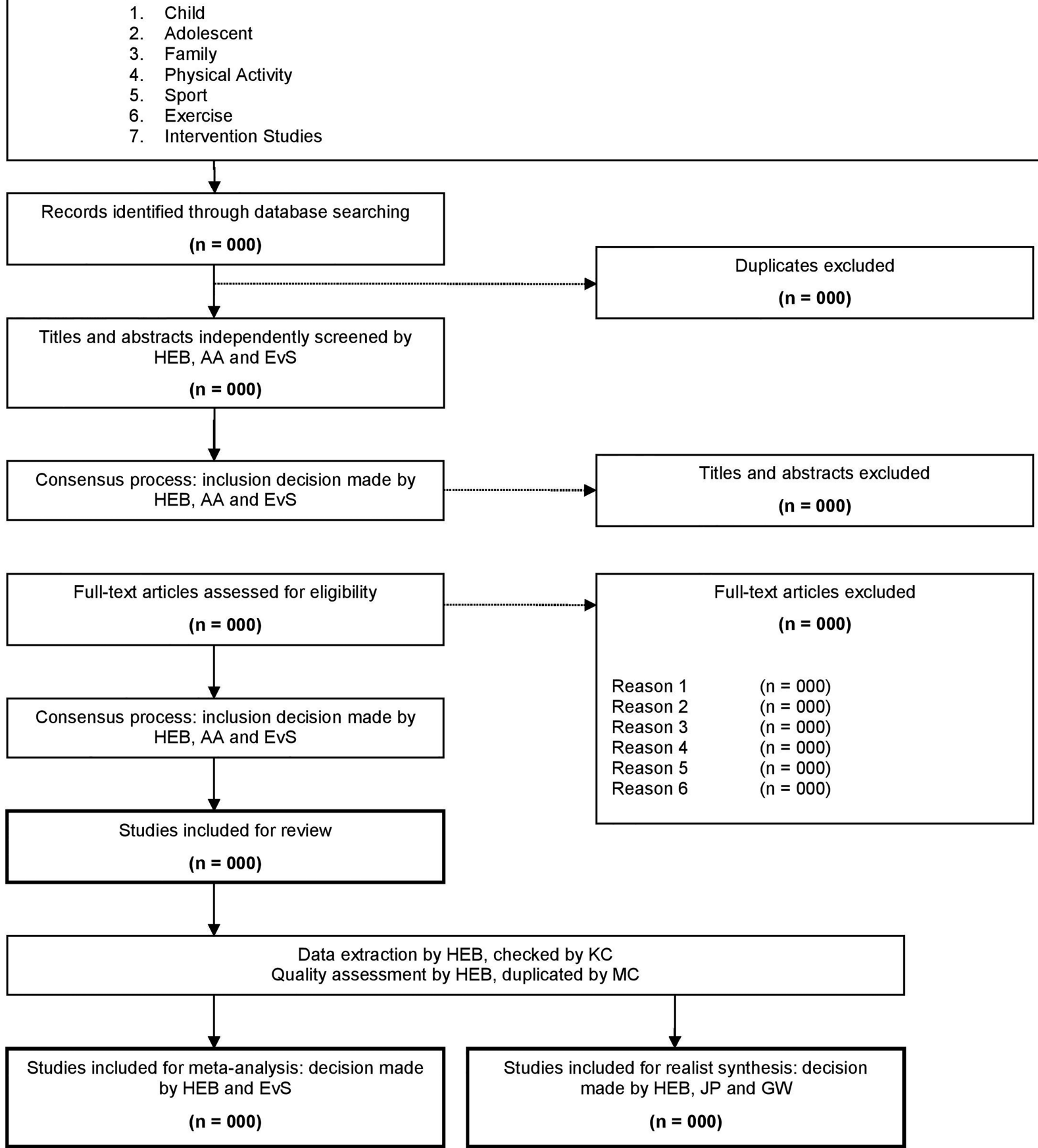

Figure 1 Search strategy for review of existing intervention 1 studies. 


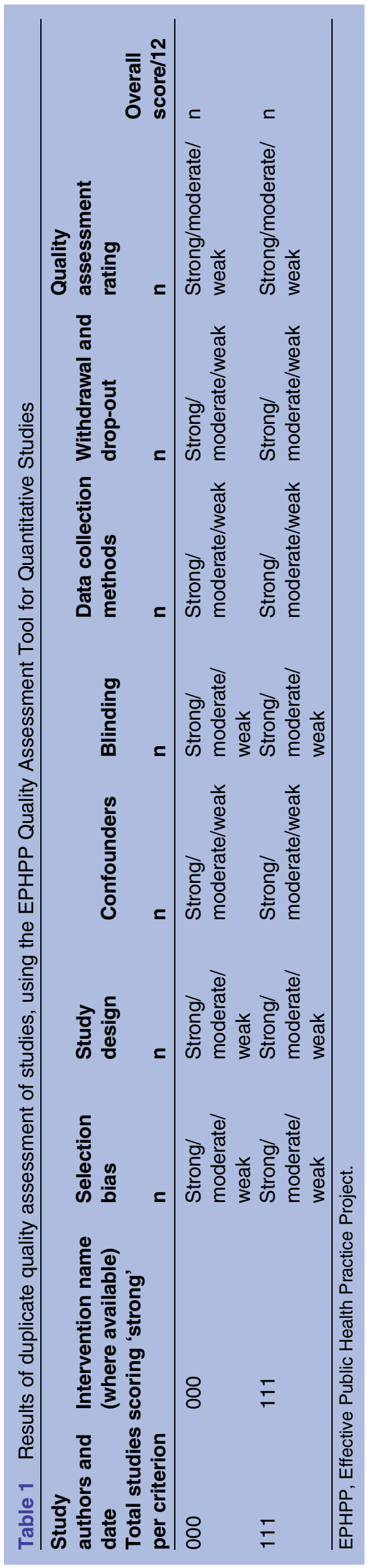

modify physical activity in any way, it will be included. Studies that engage the family as one of a range of settings, and that isolate the effect by evaluating the family-only intervention separately, will be included. Studies that do not have a substantive role for families in the physical activity component (ie, parent newsletters only) will be excluded. Studies that recruited samples who were overweight or from the general population (ie, whole school or community) will be included, but those in which participants were defined as 'obese' (by whichever definition the authors put forward) will be excluded. Valid measures of physical activity (either as total activity, or during specific periods of the day or week) may include those measured in free living by self-report or proxy-report questionnaire or diary, pedometer, accelerometer, inclinometer or heart rate monitor. These exclusion criteria are definitive, to ensure search results are reflective of specific review objectives. Results of the duplicate screening will be reported using figure 1 .

Those studies eligible for general review may also be included in the meta-analysis, the realist synthesis, or both. This will result in three separate but overlapping groups of interventions: (1) studies for general review, (2) studies for realist synthesis and (3) studies for meta-analysis. Studies that report detailed intervention descriptions, or that explore intervention mechanisms (either in analysis or discussion), will be included in the realist synthesis. Studies reporting intervention effect on physical activity (with mean, SD and number of participants analysed), in both intervention and control groups, will be included in the meta-analysis. Those studies that do not meet specific inclusion criteria for either realist synthesis or meta-analysis will be included in a narrative summary of the literature.

\section{Quality assessment}

Quality assessment will be conducted by the lead author using the Effective Public Health Practice Project Quality Assessment Tool for Quantitative Studies; this will be duplicated by an additional author (MJMC) and inter-rater reliability reported as a percentage of items without initial consensus. The Effective Public Health Practice Project tool rates studies as 'strong', 'moderate' or 'weak', using six scales (selection bias, study design, confounders, blinding, data collection methods and withdrawals and drop-outs). Studies are then rated to give an aggregate overall score of 'strong', 'moderate' or 'weak' ('strong' if no 'weak' individual-scale ratings are designated, 'moderate' if one, and 'weak' if two or more). The tool has been recommended for use in assessing public health interventions based on acceptable content and construct validity. ${ }^{25}$ Quality assessment results will be reported using table 1 .

\section{Data extraction}

Key data regarding study participants, intervention setting and characteristics, and outcomes will be extracted by the primary author (HEB), and checked 
for accuracy by another author (KC). Discrepancies will be resolved through consensus discussion. Descriptive data on all studies will be reported using table 2 (table headings detail the information to be extracted).

\section{REALIST SYNTHESIS}

Procedures for the realist synthesis will be informed by the output of the RAMESES Project, which collated expert input and evidence review into comprehensive guidance and publication standards. ${ }^{24}$ The primary function of the realist synthesis will be to attempt to explain the outcome patterns observed in included studies. This analysis will address the key realist questions; "what works for whom, under what circumstances, how, and why?"

An initial programme theory will first be developed through a consensus process facilitated by GW, utilising the content expertise of all authors. This programme theory will describe the context and mechanisms necessary to elicit a specified outcome (namely increased physical activity in children). The inclusion of data to inform programme theory development will be guided by the principles of 'relevance' and 'rigour' (as identified by RAMESES output standards, ${ }^{24}{ }^{26}$ ) will then be extracted and coded using nVivo qualitative analysis software (first by one author (HEB); and then checked by a second author (JP)). For each 'stage' (ie, text box, or connecting arrow) of the theory, inferences will be made about the possible realist explanation (ie, the context within which change may be triggered, or the mechanisms which precede change). We will also consider how context, mechanism and outcome may interact for specific intervention strategies (eg, goal-setting). Where necessary, supplementary data will be accessed (eg, adding process evaluation or protocol papers of included studies).

Data extracted will be mapped against the initial programme theory to identify areas of strength, and areas that require further research. The programme theory will then be refined to reflect mechanisms that are supported by evidence (eg, arrow thickness may be used to reflect the relative strength of the evidence, and dashed connecting lines may indicate hypothesised configurations of context, mechanism and outcome). If appropriate, existing substantive theory to corroborate stages of the programme theory will be sought.

\section{META-ANALYSIS}

Additional data (specific information for total physical activity, intensity-specific physical activity or domainspecific physical activity) will be extracted for meta-analysis, checked for accuracy by a second author (KC). The type of data extracted (eg, relevant model statistics, coefficients, between-group mean differences, within-group means) will differ according to the data reported in each paper. We will also collect the corresponding measures of precision (eg, SDs, SEs, 95\% CIs) and number of participants analysed. Each study will contribute one overall effect size, and we will extract information on potential moderators (eg, studies stratified by sex, ethnicity, weight status) for later subgroup analyses where possible.

To account for the differing unit of measurement we anticipate across studies, we will calculate standardised effect sizes. Intervention effects for each study will likely be represented by the standardised mean difference in outcome, calculated as the physical activity level at follow-up adjusted for physical activity level at baseline (or mean activity change from baseline) divided by the pooled SD of change in physical activity from baseline. Effect sizes for all included studies will then be combined using a random-effects model to derive an overall summary (average) effect estimate (and 95\% CI) and assessed against previously used criteria (ie, overall standardised mean difference of -0.2 is considered small, -0.5 moderate and -0.8 large. ${ }^{27}$ ). Predefined subgroup analyses will be conducted (age, sex, ethnicity and weight status), and issues of outlier and publication bias considered. All computation and analysis of data will be conducted using STATA software by one author (HEB), in collaboration with a second author (EvS). Results of the meta-analysis will be reported using a forest plot of the overall standardised mean difference.

Table 2 Descriptive characteristics of included studies

\begin{tabular}{|c|c|c|c|c|c|c|c|}
\hline $\begin{array}{l}\text { Study (first } \\
\text { author; year } \\
\text { of } \\
\text { publication; } \\
\text { country) }\end{array}$ & $\begin{array}{l}\text { Design (study } \\
\text { design; level of } \\
\text { randomisation } \\
\text { where } \\
\text { applicable) }\end{array}$ & $\begin{array}{l}\text { Participants } \\
\text { ( } \mathrm{n} \text {, children } \\
\text { analysed; } \\
\text { mean years } \\
\text { of age } \pm S D \text { at } \\
\text { baseline; } \% \\
\text { male; mean } \\
\text { BMl } \pm \text { SD at } \\
\text { baseline) }\end{array}$ & $\begin{array}{l}\text { Physical } \\
\text { activity } \\
\text { measurement } \\
\text { (assessment } \\
\text { period; } \\
\text { measure used; } \\
\text { outcomes } \\
\text { reported) }\end{array}$ & $\begin{array}{l}\text { Intervention } \\
\text { (intervention } \\
\text { name; } \\
\text { description) }\end{array}$ & $\begin{array}{l}\text { Intervention } \\
\text { (intervention } \\
\text { duration; } \\
\text { delivery; } \\
\text { theoretical } \\
\text { grounding) }\end{array}$ & $\begin{array}{l}\text { Control } \\
\text { (where } \\
\text { applicable, } \\
\text { description } \\
\text { of any } \\
\text { procedure) }\end{array}$ & $\begin{array}{l}\text { Outcome } \\
\text { (PA } \\
\text { change } \\
\text { and } \\
\text { statistical } \\
\text { tests } \\
\text { reported) }\end{array}$ \\
\hline 000 & 000 & 000 & 000 & 000 & 000 & 000 & 000 \\
\hline 111 & 111 & 111 & 111 & 111 & 111 & 111 & 111 \\
\hline
\end{tabular}




\section{DISCUSSION}

This review will be the first to use the framework of a traditional review to conduct a dual meta-analysis and realist synthesis. This combined approach will offer both a quantitative and a qualitative exploration of the available evidence. Each method answers the criticism of the other; a rigorous meta-analysis will provide a numerical evaluation of intervention efficacy, while a wellconducted realist synthesis will explore study context and mechanisms. This innovative method will therefore comprehensively examine interventions that explicitly engage the family unit to increase physical activity in children. The meta-analysis may also identify specific subgroups for whom interventions are most effective, and will offer an integrated summary of available evidence. The realist synthesis will contribute to understanding factors that may mediate or moderate intervention outcomes, further informing the development of robust interventions. This may subsequently direct policymakers and practitioners towards actions that are likely to bring about positive behaviour change.

In addition to offering a more exhaustive assessment of published studies, this review will supersede existing reviews ${ }^{14}{ }^{18-20}$ by including substantially more interventions. A brief scoping review identified a number of interventions published within the past 5 years, indicating the need for an update in this field. Exploring the most recent evidence available, particularly given the advances in physical activity measurement ${ }^{28}$ and the application of new technologies in changing behaviour (eg, study material delivered through mobile phones or tablets), ${ }^{29}$ will provide a contemporary summary of effective techniques. Such synthesis will then inform future work aimed at improving and maintaining physical activity levels at the critical juncture before children progress to secondary school.

\section{Potential limitations}

The proposed work may have several limitations. As in previous reviews, ${ }^{18} 20$ we will be reliant on peer-reviewed published data (rather than including grey literature). While this will ensure a focused, and therefore manageable, number of studies are reviewed, it does make the review vulnerable to publication bias. ${ }^{30} 31$ Additionally, we have not built study author contact into our review protocol. Given the expected range of study dates of publication (ie, previous reviews have included relevant interventions published as early as 1970), we anticipate difficulties in obtaining the data. While we acknowledge the aforementioned publication bias, in reviewing only published information we avoid skewing the results in favour of those studies conducted most recently.

\section{Dissemination}

The results of this study will be disseminated to academic and non-academic audiences through peerreviewed publications, conferences, formal presentations to policy makers and practitioners and in formal stakeholder meetings. Evidence generated from this synthesis will also be used to inform the development of theory-driven, evidence-based interventions aimed at engaging the family to increase physical activity levels in children.

Contributors HEB was responsible for conducting and coordinating aspects of this study. All authors contributed considerably to editing of all written work. Article screening, general literature analyses and extraction and interpretation of data were carried out by HEB, AJA, EvS and KC. HEB and MJMC carried out the quality assessment of included studies. The development of the programme theory was done by all authors. HEB, JP and GW were involved in coding, extraction of mechanisms, and realist synthesis and interpretation of the study. HEB and EvS conducted meta-analysis of quantitative data.

Competing interests None.

Provenance and peer review Not commissioned; externally peer reviewed.

Open Access This is an Open Access article distributed in accordance with the terms of the Creative Commons Attribution (CC BY 4.0) license, which permits others to distribute, remix, adapt and build upon this work, for commercial use, provided the original work is properly cited. See: http:// creativecommons.org/licenses/by/4.0/

\section{REFERENCES}

1. Ekelund U, Luan J, Sherar LB, et al. Moderate to vigorous physical activity and sedentary time and cardiometabolic risk factors in children and adolescents. JAMA 2012;307:704-12.

2. Andersen LB, Riddoch C, Kriemler S, et al. Physical activity and cardiovascular risk factors in children. $\mathrm{Br} J$ Sports Med 2011;45:871-6.

3. Hills AP, Andersen LB, Byrne NM. Physical activity and obesity in children. Br J Sports Med 2011;45:866-70.

4. Wilks DC, Sharp SJ, Ekelund U, et al. Objectively measured physical activity and fat mass in children: a bias-adjusted meta-analysis of prospective studies. PLOS ONE 2011;6:e17205.

5. Boreham CA, McKay HA. Physical activity in childhood and bone health. Br J Sports Med 2011;45:877-9.

6. Biddle SJ, Asare M. Physical activity and mental health in children and adolescents: a review of reviews. Br J Sports Med 2011;45:886-95.

7. Janssen I, Leblanc AG. Systematic review of the health benefits of physical activity and fitness in school-aged children and youth. Int J Behav Nutr Phys Act 2010;7:40.

8. Andersen LB, Harro M, Sardinha LB, et al. Physical activity and clustered cardiovascular risk in children: a cross-sectional study (The European Youth Heart Study). Lancet 2006;368:299-304.

9. Nader PR, Bradley $\mathrm{RH}$, Houts RM, et al. Moderate-to-vigorous physical activity from ages 9 to 15 years. JAMA 2008;300:295-305.

10. Dumith SC, Gigante DP, Domingues MR, et al. Physical activity change during adolescence: a systematic review and a pooled analysis. Int J Epidemiol 2011;40:685-98.

11. Corder K, Sharp SJ, Atkin AJ, et al. Change in objectively measured physical activity during the transition to adolescence.

Br J Sports Med Published online first: 9 Apr 2014. doi:10.1136/ bjsports-2013-093190

12. Jago R. Commentary: Age-related decline in physical activity during adolescence-an opportunity to reflect on intervention design and key research gaps. Int $J$ Epidemiol 2011;40:699-700.

13. Metcalf B, Henley W, Wilkin T. Effectiveness of intervention on physical activity of children: systematic review and meta-analysis of controlled trials with objectively measured outcomes (EarlyBird 54). BMJ 2012;345:e5888.

14. van Sluijs EM, McMinn AM, Griffin SJ. Effectiveness of interventions to promote physical activity in children and adolescents: systematic review of controlled trials. BMJ 2007;335:703.

15. NICE. Promoting physical activity for children and young people: guidance. National Institute for Health and Clinical Excellence, 2009.

16. Activity, N.P.H.C.C.P. Intervention review: family and community. National Institute for Health and Clinical Excellence, 2008.

17. Sallis JF, Prochaska JJ, Taylor WC. A review of correlates of physical activity of children and adolescents. Med Sci Sports Exerc 2000;32:963-75. 
18. Salmon J, Booth ML, Phongsavan $\mathrm{P}$, et al. Promoting physical activity participation among children and adolescents. Epidemiol Rev 2007;29:144-59.

19. O'Connor TM, Jago R, Baranowski T. Engaging parents to increase youth physical activity a systematic review. Am J Prev Med 2009;37:141-9.

20. van Sluijs EM, Kriemler S, McMinn AM. The effect of community and family interventions on young people's physical activity levels: a review of reviews and updated systematic review. Br J Sports Med 2011;45:914-22.

21. Oakley A, Strange V, Bonell C, et al. Process evaluation in randomised controlled trials of complex interventions. $B M J$ 2006;332:413-16.

22. Egger M, Smith GD. Meta-analysis. Potentials and promise. BMJ 1997;315:1371-4.

23. Riley RD, Higgins JP, Deeks JJ. Interpretation of random effects meta-analyses. BMJ 2011;342:d549.
24. Wong G, Greenhalgh T, Westhorp G, et al. RAMESES publication standards: realist syntheses. BMC Med 2013;11:21.

25. Deeks JJ, Dinnes J, D'Amico R, et al. Evaluating non-randomised intervention studies. Health Technol Assess 2003;7:iii-x. 1-173.

26. Pawson R. A realist perspective. Sage Publications, 2006.

27. Cohen J. Statistical power analysis for the behavioral sciences. Psychology Press, 1988.

28. Matthews CE, Hagströmer M, Pober DM, et al. Best practices for using physical activity monitors in population-based research. Med Sci Sports Exerc 2012;44(Suppl 1):S68-76.

29. Bort-Roig J, Gilson ND, Puig-Ribera A, et al. Measuring and influencing physical activity with smartphone technology: a systematic review. Sports Med 2014;44:671-86.

30. Dickersin $\mathrm{K}$. The existence of publication bias and risk factors for its occurrence. JAMA 1990;263:1385-9.

31. Easterbrook PJ, Berlin JA, Gopalan R, et al. Publication bias in clinical research. Lancet 1991;337:867-72. 